\title{
Differential involvement of the medial prefrontal cortex across variants of contextual fear conditioning
}

\author{
Nicholas A. Heroux, Patrese A. Robinson-Drummer, Hollie R. Sanders, Jeffrey B. Rosen, \\ and Mark E. Stanton \\ Department of Psychological and Brain Sciences, University of Delaware, Newark, Delaware 19716, USA
}

\begin{abstract}
The context preexposure facilitation effect (CPFE) is a contextual fear conditioning paradigm in which learning about the context, acquiring the context-shock association, and retrieving/ expressing contextual fear are temporally dissociated into three distinct phases. In contrast, learning about the context and the context-shock association happens concurrently in standard contextual fear conditioning (sCFC). By infusing the $\mathrm{GABA}_{\mathrm{A}}$ receptor agonist muscimol into medial prefrontal cortex ( $\mathrm{mPFC}$ ) in adolescent Long-Evans rats, the current set of experiments examined the functional role of the mPFC in each phase of the CPFE and sCFC. In the CPFE, the mPFC is necessary for the following: acquisition and/or consolidation of context memory (Experiment 1), reconsolidation of a context memory to include shock (Experiment 2), and expression of contextual fear memory during a retention test (Experiment 3). In contrast to the CPFE, inactivation of the MPFC prior to conditioning in sCFC has no effect on acquisition, consolidation, or retention of a contextual fear memory (Experiment 4). Interestingly, the $\mathrm{mPFC}$ is not required for acquiring a context-shock association (measured by post-shock freezing) in the CPFE or sCFC (Experiment $2 \mathrm{~b}$ and 4). Taken together, these results indicate that the $\mathrm{mPFC}$ is differentially recruited across stages of learning and variants of contextual fear conditioning (CPFE versus sCFC). More specifically, separating out learning about the context and the context-shock association necessitates activation of the medial prefrontal cortex during early learning and/or consolidation.
\end{abstract}

It is a widely accepted view that multiple neural systems support different aspects of learning and memory in Pavlovian fear conditioning (Fanselow and Poulos 2005; Giustino and Maren 2015; Jin and Maren 2015; Tovote et al. 2015). Key brain structures supporting contextual fear conditioning include the hippocampus, amygdala, and the medial prefrontal cortex (mPFC). While the roles of the hippocampus and amygdala have been largely attributed to context and context-shock learning, respectively, the specific role of the $\mathrm{mPFC}$ in contextual fear conditioning remains poorly understood.

In standard contextual fear conditioning (sCFC), animals learn about the context and acquire the context-shock association concurrently. Contextual fear learning can then be probed by measuring a species-typical freezing response in a post-shock test occurring immediately after conditioning or in a delayed retention test (Fanselow 1980, 1990). Contextual fear learning in this procedure is supported by a dominant hippocampal system in which the features of the context are bound into a conjunctive representation, or, when the hippocampal system is dysfunctional, by a neocortical system that processes individual features of the context (Rudy and O'Reilly 2001; Rudy et al. 2004; Rudy 2009; Wiltgen et al. 2006). In a variant of contextual fear conditioning called the context preexposure facilitation effect (CPFE), acquiring a context representation, acquiring the context-shock association, and the expression of contextual fear memory occur in three distinct phases usually separated by $24 \mathrm{~h}$. In contrast to sCFC, the CPFE requires the hippocampus during each phase of learning and cannot be supported by the neocortical feature-based system (Rudy and O'Reilly 2001; Matus-Amat et al. 2004; Rudy 2009; Jablonski et al. 2012). The amygdala is required for the acquisition of the context-shock association in both sCFC and the CPFE (Kim et al.

\section{Corresponding author: Stanton@psych.udel.edu}

Article is online at http://www.learnmem.org/cgi/doi/10.1101//m.045286. 117.
1993; Maren et al. 1996; Malkani and Rosen 2001; Malkani et al. 2004; Matus-Amat et al. 2007).

The role of the mPFC in fear conditioning has been traditionally attributed to the long-term, systems-level consolidation of memory and to the regulation of fear memory expression via the prelimbic (PL) and infralimbic (IL) sub-regions (Frankland and Bontempi 2005; Kitamura and Inokuchi 2014; Giustino and Maren 2015; Jin and Maren 2015). Systems consolidation theory holds that early encoding, storage, and retrieval of a contextual fear memory depends primarily on hippocampal-amygdala circuitry and gradually reorganizes to rely on neocortical circuits, including the medial prefrontal cortex, for remote memory retrieval and maintenance (Squire and Alvarez 1995; Frankland and Bontempi 2005; Frankland et al. 2006; Wang and Morris 2010; Bero et al. 2014; Kitamura and Inokuchi 2014). Recent research has suggested a role of the mPFC in processing contextual information during training in trace and contextual fear conditioning (Gilmartin et al. 2012, 2013; Zelikowsky et al. 2014). Taken together with demonstrations of contextual fear conditioning driving gene expression in the mPFC (Asok et al. 2013; Schreiber et al. 2014; Chakraborty et al. 2016), these studies suggest an active role of the mPFC in contextual fear acquisition.

The current study examined adolescent rats (PD31) to help guide future research across earlier (e.g., juvenile) and later (e.g., adult) stages of ontogeny. Our laboratory has previously shown that the CPFE develops between postnatal day (PD) 17 and 24 in the rat (Schiffino et al. 2011). Interestingly, PD17 rats are able to acquire a context-shock association in SCFC and express fear in a

\footnotetext{
(C) 2017 Heroux et al. This article is distributed exclusively by Cold Spring Harbor Laboratory Press for the first 12 months after the full-issue publication date (see http://learnmem.cshlp.org/site/misc/terms.xhtml). After 12 months, it is available under a Creative Commons License (Attribution-NonCommercial 4.0 International), as described at http://creativecommons.org/licenses/by-nc/ $4.0 /$.
} 
post-shock freezing test, but lack the ability to consolidate a stable contextual fear memory until later stages of ontogeny (around PD24) (Rudy and Morledge 1994). Accordingly, the inability of PD17 rats to acquire contextual fear in the CPFE likely reflects an inability to acquire or consolidate a conjunctive context representation on the preexposure day of the CPFE, as PD17 rats do not demonstrate post-shock freezing on the training day of the CPFE (Jablonski et al. 2012). We have previously shown that both sCFC and the CPFE induce expression of the immediate early gene (IEG) early growth response gene-1 (Egr-1) in the medial prefrontal cortex of adolescent (PD31) and adult rats (PD60) (Asok et al. 2013; Schreiber et al. 2014; Chakraborty et al. 2016). Despite this, the functional role of the $\mathrm{MPFC}$ in the early stages of encoding and memory formation during context and contextual fear learning is poorly understood at any age in the rat. The present study begins to examine this issue in early adolescent (PD31) rats.

The current set of experiments examined both variants of contextual fear conditioning (CPFE and sCFC) in an effort to characterize the role of the mPFC in context learning, contextual fear acquisition, and expression of contextual fear memory. This was achieved by reversibly inactivating the $\mathrm{MPFC}$ via intra-mPFC infusions of the $\mathrm{GABA}_{\mathrm{A}}$ receptor agonist muscimol prior to each phase of the CPFE ("Experiments 1-3") and prior to SCFC training ("Experiment 4"). The current set of experiments provides four main findings. We provide the first demonstration, at any age in the rat, that the mPFC is required for the acquisition and/or consolidation of a conjunctive context representation during incidental context learning (i.e., the preexposure day of the CPFE) in the absence of aversive stimuli ("Experiment 1"). Second, we demonstrate a role of the $\mathrm{mPFC}$ in updating a context memory to include shock in the CPFE ("Experiment 2"). Third, we show a differential involvement of the mPFC in contextual fear expression across stages of contextual fear conditioning, with the mPFC being required during a retention test 24 -h after fear conditioning, but not post-shock freezing in the CPFE (i.e., immediately after conditioning; "Experiments 2-4"). Finally, we demonstrate that the $\mathrm{mPFC}$ is not required for short-term acquisition of a context-shock association in either the CPFE or SCFC, or consolidation of long-term fear memory in sCFC ("Experiments 2 and 4").

\section{Results}

Experiment 1: inactivation of the mPFC prior to context preexposure in the CPFE impairs the acquisition and/or consolidation of a context representation

In this study, muscimol or PBS was infused into mPFC (Fig. 1) $15 \mathrm{~min}$ before preexposure to the training context (or an alternate control context, Alt-Pre), and no infusions occurred prior to immediate-shock training or the $5 \mathrm{~min}$ retention test (Fig. 2). Analyses for Experiment 1 were run on 35 animals distributed across the following groups: PBS $(n=12)$, Muscimol $(n=11)$, and Pooled-Alt-Pre $(n=14$, pooled across drug, see Materials and Methods). Retention test freezing analyzed with a one-way ANOVA (Drug; PBS, Muscimol,
Pooled-Alt-Pre) revealed a significant main effect of Drug $\left[F_{(2,34)}\right.$ $=23.06, P<0.0001]$. Freezing behavior for the Muscimol group was significantly disrupted compared with animals infused with PBS $(P=0.0001)$ but did not significantly differ from the Pooled-Alt-Pre control group $(P=0.26)$. These results indicate that inactivation of the mPFC prior to context preexposure abolishes the acquisition and/or consolidation of a conjunctive context representation (see General discussion).

\section{Experiment 2: inactivation of the mPFC prior to context-shock training in the CPFE spares acquisition but impairs $24 \mathrm{~h}$ retention \\ of contextual fear}

In this experiment, mPFC infusions occurred only prior to immediate-shock training and freezing was measured only during the retention test (Experiment 2a) or during a post-shock period on the training day and again on the retention test day (Experiment 2b; Fig. 3). Analyses for Experiment 2a (Fig. 3, left panel) were run on 37 animals distributed across the following groups: PBS $(n=12)$, Muscimol $(n=13)$, and Pooled-Alt-Pre $(n=12)$. Freezing behavior for a 5-min retention test occurring $24 \mathrm{~h}$ after immediate shock training (with no post-shock test) was analyzed with a oneway ANOVA (Drug; PBS, Muscimol, Pooled-Alt-Pre). ANOVA revealed a significant main effect of Drug $\left[F_{(2,34)}=16.48, P<\right.$ $0.0001]$. Freezing behavior in the Muscimol group was significantly disrupted relative to animals infused with PBS $(P=0.0001)$ and did not significantly differ from the Pooled-Alt-Pre control group $(P=0.10)$. The PBS group differed significantly from Pooled-Alt-Pre $(P=0.0001)$.

Analyses for Experiment 2b (Fig. 3, right panel) were run on 33 animals distributed across the following groups: PBS $(n=11)$, Muscimol $(n=12)$, and Pooled-Alt-Pre $(n=10)$. Freezing behavior for a 3-min post-shock test immediately after context-shock training and a within-subjects 5 -min retention test $24 \mathrm{~h}$ later was analyzed with a Drug (PBS, Muscimol, Pooled-Alt-Pre) $\times$ Phase

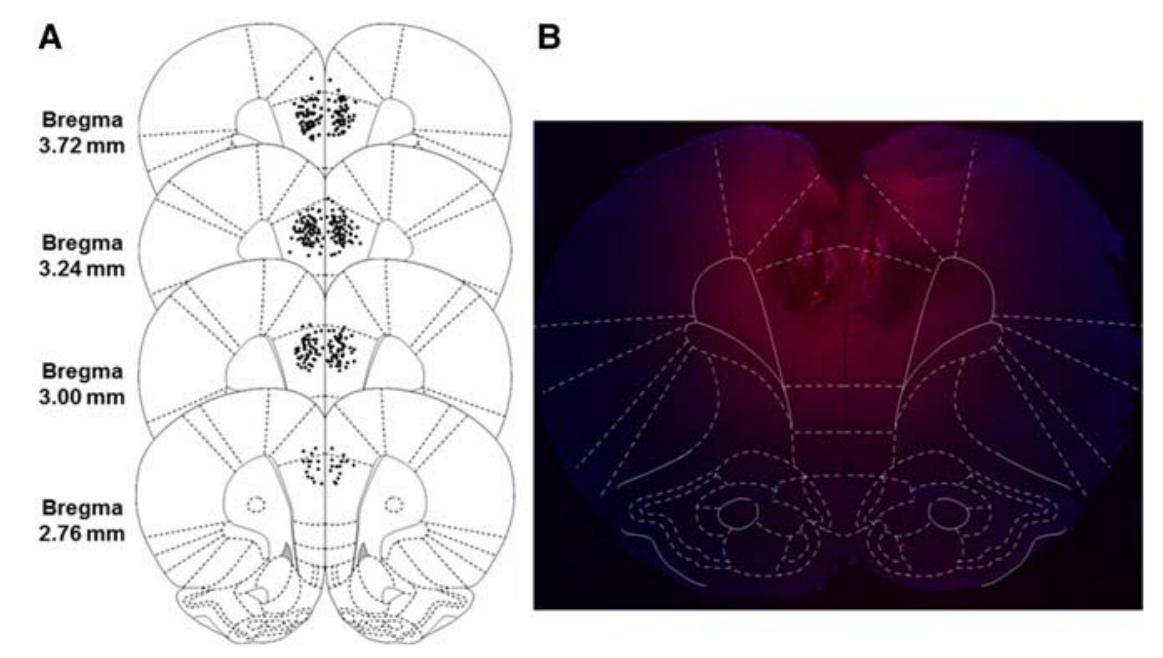

Figure 1. Schematic representation of the majority of injection cannula tip placements in the mPFC for Experiments 1-4 (left, $A$ ) with visualization of the drug spread using the fluorescent muscimol BODIPY-TMRX (right, $B$ ). (A) Animals included in final analyses are represented by filled black dots. Placements ranged from 4.20 to $2.52 \mathrm{~mm}$ from bregma, with cannula placements from 10 animals being excluded for misses either anterior or posterior to this range (not shown). (B) Image taken from an animal infused with fluorescent muscimol into the mPFC, overlaid with a digital mPFC plate to examine the dorsal-ventral and medial-lateral drug spread. The anterior-posterior spread (not shown) was analyzed in sagittal sections and ranges from 1.75 to $2 \mathrm{~mm}$ from the cannula tract in any direction. (Reprinted from Paxinos and Watson 2007 with permission from Elsevier (C) 2007.) 


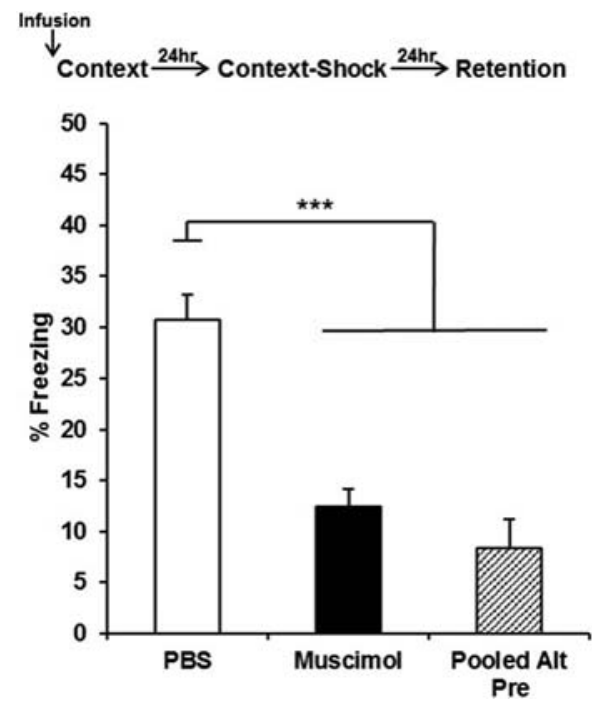

Figure 2. Mean percent freezing $( \pm S E M)$ on the retention test day as a function of drug and behavioral treatment group in Experiment 1. Infusion of muscimol into the mPFC prior to context preexposure on Day 1 (followed by immediate-shock training in the same context on Day 2) reduced freezing during a retention test on Day 3 to a level comparable to nonassociative controls preexposed to an alternate context on Day 1 (pooled across drug treatment) and significantly disrupted freezing relative to animals infused with PBS on the preexposure day. $\left({ }^{* *}\right) P<0.001$.

(Post-shock, Retention) repeated-measures ANOVA with phase being the repeated measure. ANOVA revealed a significant main effect of Drug $\left[F_{(2,27)}=13.52, P<0.0001\right]$ and Phase $\left[F_{(1,27)}=8.17, P=\right.$ $0.008]$ in addition to a significant Drug $\times$ Phase interaction $\left[F_{(2,27)}\right.$ $=3.83, P=0.034]$. There was no significant difference between the Muscimol and PBS groups measured by freezing behavior in a 3-min post-shock test $(P=0.54)$, with both groups freezing significantly higher than the Pooled-Alt-Pre control group $(P s<0.01)$. In contrast, freezing behavior during a $24 \mathrm{~h}$ retention test was significantly disrupted in the Muscimol group relative to animals infused with PBS $(P=0.006)$, replicating the disrupted retention seen in Experiment 2a. There was no significant difference between freezing behavior in the post-shock or 24-h retention test in the PBS group $(P=0.83)$. Taken together, these results indicate that inactivation of the mPFC prior to context-shock training impairs the 24 $\mathrm{h}$ retention of contextual fear but leaves acquisition intact.

\section{Experiment 3: inactivation of the mPFC prior to a 24-h retention test in the CPFE impairs contextual fear expression}

In this experiment, mPFC infusions occurred only prior to the 24-h retention test in the CPFE (Fig. 4). Analyses for Experiment 3 were run on 35 animals distributed across the following groups: PBS $(n=11)$, Muscimol $(n=12)$, and Pooled-Alt-Pre $(n=12)$. Retention test freezing analyzed with a one-way ANOVA (Drug; PBS, Muscimol, Pooled-Alt-Pre) revealed a significant main effect of Drug $\left[F_{(2,32)}=7.96, P=0.001\right]$. Freezing behavior for the Muscimol group was significantly disrupted compared with animals infused with PBS $(P=0.002)$ but did not significantly differ from the Pooled-Alt-Pre control group $(P=0.80)$. These results suggest that inactivation of the $\mathrm{mPFC}$ prior to retention testing disrupts the expression of the context-shock association acquired $24 \mathrm{~h}$ prior (see General discussion).
Experiment 4: inactivation of the $\mathrm{mPFC}$ prior to sCFC has no effect on contextual fear acquisition (post-shock freezing) or $24 \mathrm{~h}$ retention

In this experiment (Fig. 5), mPFC infusions occurred prior to standard contextual fear conditioning involving 3 min of context exposure terminating with two footshocks, with half of the animals receiving a 3-min post-shock freezing test (Fig. 5, Panel A) and the other half receiving a 24-h retention test with no postshock period (Fig. 5, Panel B). Animals receiving two immediate shocks terminating with a post-shock or a 24 -h retention freezing test served as nonassociative controls. Analyses for Experiment 4 were run on 62 animals distributed across the following groups: Imm-Shock-Post-shock-PBS $(n=4)$, Imm-Shock-Post-shockMuscimol $(n=4)$, Imm-Shock-Ret-PBS $(n=5)$, Imm-Shock-RetMuscimol $(n=5)$, Post-shock-PBS $(n=11)$, Post-shock-Muscimol $(n=12)$, Retention-PBS $(n=10)$, and Retention-Muscimol $(n=11)$. Freezing behavior analyzed with a factorial ANOVA (Drug [PBS, Muscimol] $\times$ Training [Imm-Shock, Delayed-Shock] $\times$ Phase [Post-shock, Retention) revealed a significant main effect of Training $\left[F_{(1,54)}=46.54, P<0.0001\right]$ and Phase $\left[F_{(1,54)}=11.64, P=\right.$ $0.001]$. ANOVA also revealed a significant Phase $\times$ Training interaction $\left[F_{(1,54)}=10.59, P=0.001\right]$, with trained post-shock animals freezing significantly higher than trained retention animals regardless of drug ( $P \mathrm{~s}<0.001$; note change in $y$-axis scale across Fig. 5A,B). There were no main effects $\left[F_{(1,54)}=0.12, P=0.73\right]$ or interactions

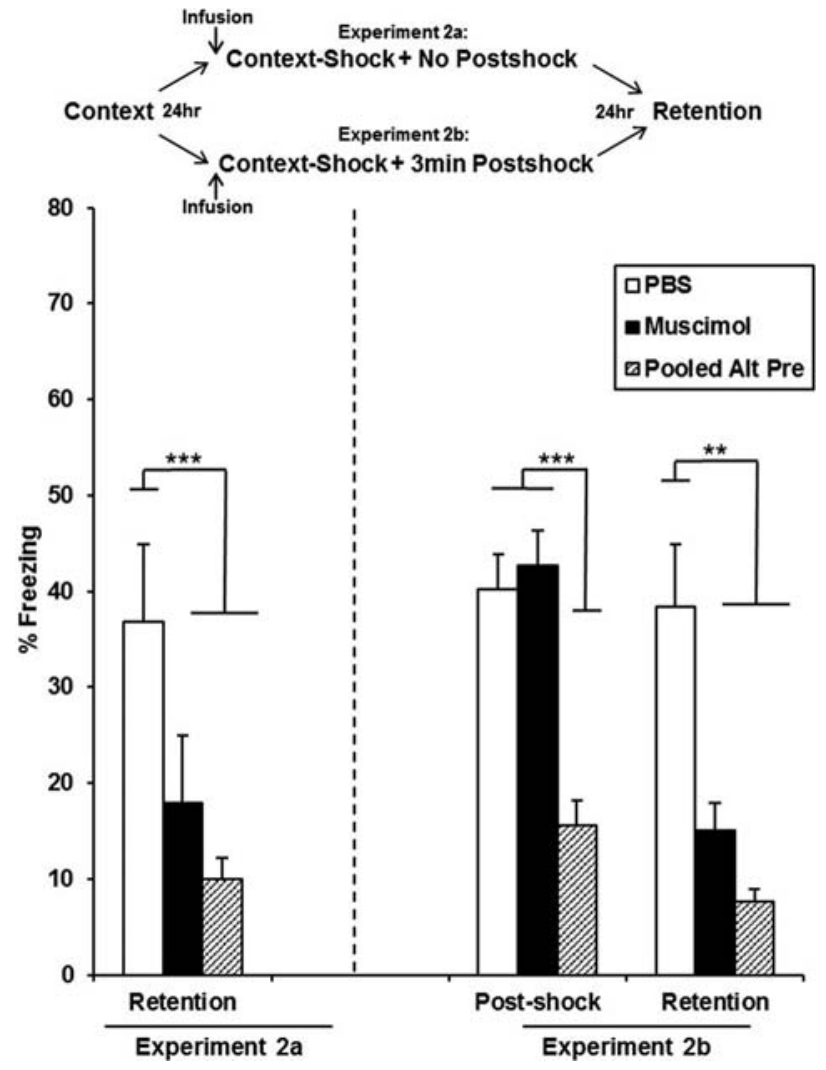

Figure 3. Mean percent freezing ( \pm SEM) in an immediate 3-min postshock or 5-min, 24-h retention test depicted for animals receiving PBS or muscimol $15 \mathrm{~min}$ prior to context-shock training. Infusion of muscimol into the mPFC prior to context-shock training had no effect on contextfear acquisition but significantly disrupted 24 -h retention test freezing relative to animals infused with PBS. This occurred regardless of whether animals received a post-shock test $24 \mathrm{~h}$ prior (Exp. 2b; right panel) or not (Exp. 2a; left panel). $\left.{ }^{\star * *}\right) P<0.001,\left({ }^{* *}\right) P<0.01$. 


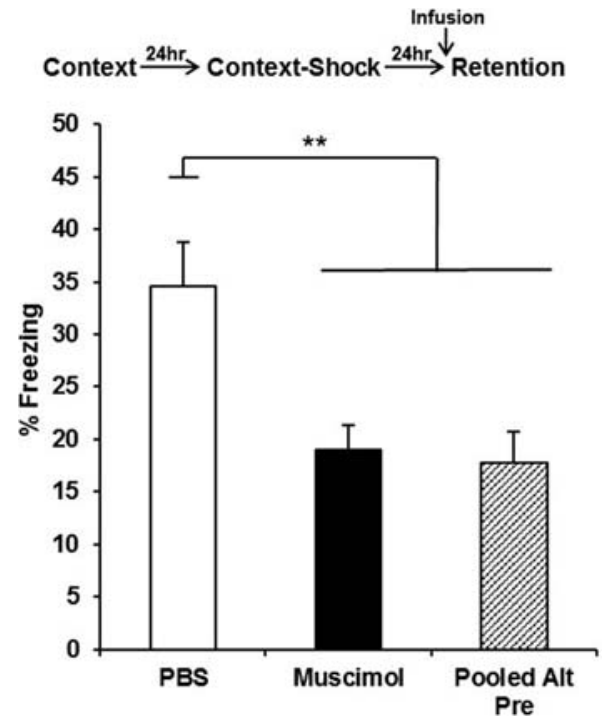

Figure 4. Mean percent freezing ( $₫$ SEM) depicted for animals receiving PBS or muscimol 15 min prior to a 5-min retention test occurring $24 \mathrm{~h}$ after conditioning with no post-shock test. Infusion of muscimol into the mPFC prior to the retention test reduced freezing to a level comparable to nonassociative controls (pooled across drug treatment) and significantly lower than animals infused with PBS $\left({ }^{* *}\right) P<0.01$.

involving the factor of Drug (Ps $>0.80$ ). Freezing during the baseline $3 \mathrm{~min}$ of context exposure prior to training (termed Preshock) was significantly lower than the trained Post-shock group $(P<0.0001)$ but not significantly different from the Imm-Shock-Post-shock-PBS or Imm-Shock-Post-shock-Muscimol groups $(P s>0.20)$. These results indicate that inactivation of the mPFC prior to conditioning in SCFC has no effect on contextual fear acquisition or retention, measured by post-shock freezing or $24 \mathrm{~h}$ retention freezing, respectively.

\section{Discussion}

The current set of experiments examined the behavioral effects of mPFC inactivation during the initial stages of encoding and memory formation across variants of contextual fear conditioning in adolescent rats. This inactivation during any phase of the CPFE—context preexposure, immediate-shock training, or testingdisrupted freezing in a 24 -h retention test. Importantly, mPFC inactivation prior to the immediate-shock training phase of the CPFE did not disrupt postshock freezing even though it abolished 24-h retention freezing in the same rats (Experiment 2b). Finally, in contrast to the CPFE, mPFC inactivation prior to delayed-shock training in sCFC had no effect on freezing either in a post-shock or 24-h retention test (Experiment 4). Whereas the current study cannot speak to the degree to which individual subregions of the mPFC contribute to these effects, our results reveal differential recruitment of the mPFC across components of learning in variants of contextual fear conditioning. In the following Discussion, we consider implications of each experiment for prefrontal function and then close with general conclusions concerning the role of prefrontal cortex in contextual fear conditioning.

To our knowledge, this is the first study to demonstrate that the mPFC is required for learning a context representation during an initial context exposure that supports subsequent association of this representation with aversive stimuli (e.g., a foot-shock; Experiment 1). The CPFE depends on the encoding of contextual cues on the preexposure day that are consolidated into a conjunctive context representation within a short temporal window following preexposure (Rudy and Wright-Hardesty 2005; Burman et al. 2009). Encoding of these features of the context and consolidation of the conjunctive representation is thought to rely on interaction between the para-hippocampal region (PHR: including entorhinal, postrhinal, and perirhinal cortices) and hippocampus (Rudy 2009). Accordingly, disrupting hippocampal functioning on the preexposure day abolishes the CPFE (Matus-Amat et al. 2004, 2007; Schiffino et al. 2011; Robinson-Drummer et al. 2016). Recent findings suggest that both the mPFC and hippocampus process contextual information in contextual fear conditioning. Both the mPFC and dHPC show increases in gene expression following contextual exposure in sCFC (Zelikowsky et al. 2014) and optogenetic silencing of mPFC excitatory neurons during fear conditioning can attenutate gene expression and activation in the hippocampal-parahippocampal system (Bero et al. 2014). Additionally, the mPFC may influence hippocampal representation of space by modulating hippocampal place fields (Hok et al. 2005, 2013; Burton et al. 2009). In conjuction with the significant disruption in contextual fear learning observed following mPFC inactivation during context learning (Experiment 1), these reports suggest the mPFC and dHPC both contribute to encoding and/or consolidating a conjunctive context representation. Furthermore,

\section{A}
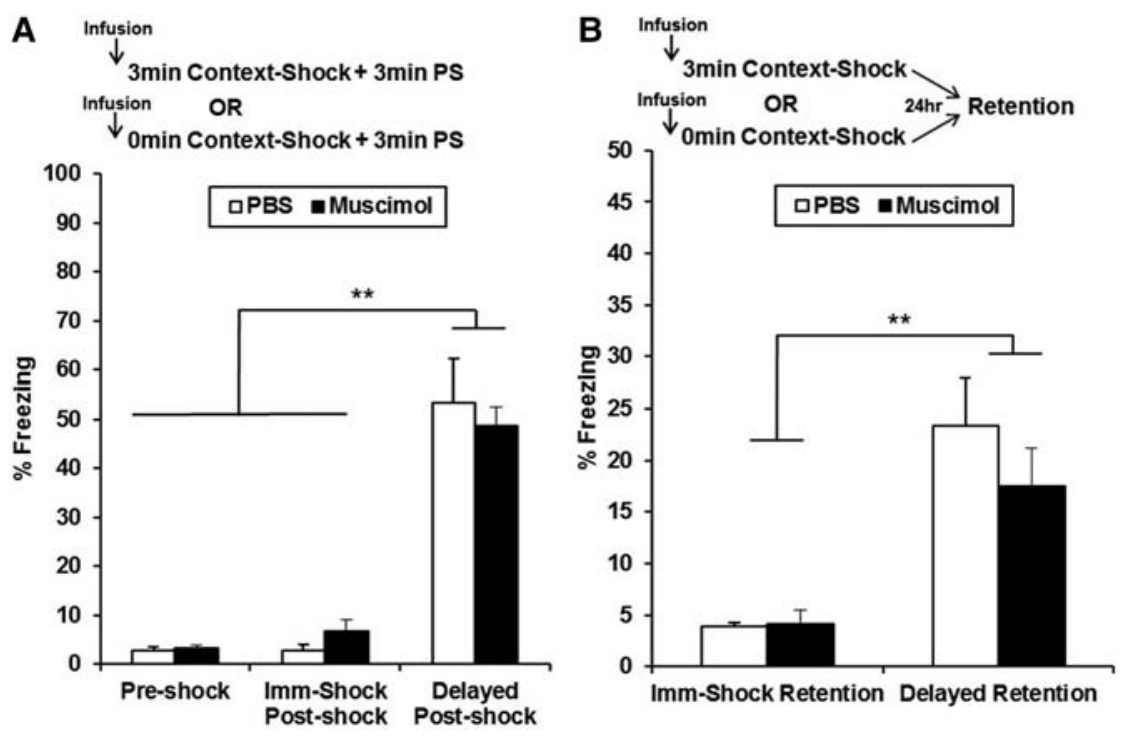

Figure 5. Mean percent freezing $( \pm S E M)$ in a between-subjects 3-min post-shock test $(A)$ or $24 \mathrm{~h}$ retention test $(B)$ depicted for animals receiving PBS or muscimol $15 \mathrm{~min}$ prior to conditioning in sCFC (note change in $y$-axis scale across panels). Animals received either $3 \mathrm{~min}$ of context exposure (Delayed-Shock condition) or no context exposure (Imm-Shock condition) prior to the two footshocks. The Preshock bar (in $A$ ) represents freezing during the 3 min of context exposure before the footshocks. Infusion of muscimol into the mPFC prior to conditioning had no effect on freezing behavior measured in the post-shock or retention tests $\left(P_{S}>0.80\right)$. Animals receiving context exposure during conditioning (Delayed-Shock) froze significantly higher than animals receiving no context exposure prior to the shock (Imm-Shock controls, Imm-Shock). $\left.{ }^{\star *}\right) P<0.01$. 
the mPFC may have a modulatory influence on the hippocampalparahippocampal system, although examining the effects of postpre-exposure mPFC inactivation alone is needed to distinguish the role of the mPFC in context encoding versus consolidation.

In Experiment 2, infusion of muscimol into the mPFC prior to immediate-shock training spared acquisition of a contextshock association (post-shock freezing) but impaired $24 \mathrm{~h}$ retention of contextual fear. The training day of the CPFE requires hippocampal-dependent pattern completion to retrieve the conjunctive context representation to be associated with an immediate-shock (Rudy 2009). The inability of mPFC inactivation to disrupt post-shock freezing suggests the MPFC plays no role in pattern completion or associating the conjunctive context representation with footshock. Intact post-shock freezing on the training day of the CPFE also rules out "performance effects" of mPFC inactivation in the present study as any deficits muscimol might produce in sensory processing (e.g., of contextual cues or shock) or in motor performance (e.g., hyperactivity) would interfere with post-shock freezing. Interestingly, unlike in SCFC, the training day of the CPFE can be viewed as the reconsolidation of a neutral context representation acquired on the previous day to be updated to include shock as a feature (Lee 2010). Accordingly, the training day of the CPFE induces the expression of Egr-1, an immediate early gene that has been linked to memory reconsolidation within the DHPC, BLA, and mPFC (Lee 2010; Maddox et al. 2011; Schreiber et al. 2014; Stern et al. 2014). Consistent with the role of the hippocampus in reconsolidation, intra-DHPC infusions of Egr-1 antisense oligodeoxynucleotide disrupt the CPFE when administered prior to immediate-shock training (Lee 2010). Such a role for Egr-1 may extend to mPFC (Asok et al. 2013). Additionally, in adult rats that underwent sCFC, inactivating the mPFC after contextual fear memory retrieval impairs subsequent memory reconsolidation and decreases the expression of Egr-1 (Einarsson and Nader 2012; Stern et al. 2014). An alternative explanation to current data is that mPFC inactivation disrupts the initial consolidation of a newly formed context-shock association in the CPFE. While plausible, inactivation of the mPFC did not disrupt the acquisition of contextual fear in either variant of contextual fear conditioning. Indeed, conditioning in both the CPFE and SCFC resulted in similarly high levels of freezing during each respective post-shock freezing test (Experiment 2 and 4). Additionally, there was no effect of mPFC inactivation on the consolidation of a context-shock association in sCFC (Experiment 4). Thus, there are no data explaining why a context-shock association, once acquired, would require the mPFC for consolidation in the CPFE but not in sCFC. Taken together with previous research, these findings suggest that inactivating the mPFC on the training day impairs the reconsolidation of neutral context memory to be updated to include shock as a feature. Additional studies are needed to further test this hypothesis.

The results of Experiment 3 indicate that inactivation of the mPFC prior to a 24-h retention test impairs the expression of contextual fear memory in the CPFE. This is in accordance with previous findings that have supported a role for the mPFC in fear memory expression (during retention) in both cue-specific and foreground contextual fear conditioning (Corcoran and Quirk 2007; Quinn et al. 2008; Laurent and Westbrook 2009; Sierra-Mercado et al. 2011). The current literature suggests that the PL and IL subregions of MPFC are important for fear expression and inhibition, respectively (Kim et al. 2009; Ji and Neugebauer 2012; Giustino and Maren 2015; Rozeske et al. 2015; Giustino et al. 2016). Accordingly, expression of learned fear results in an increase in spontaneous firing rates in PL neurons coupled with a suppression in spontaneous firing rates in IL neurons (Giustino et al. 2016). Here, we demonstrate that full inactivation of the mPFC (ACC, PL, and IL) results in a disruption in fear memory expression in a 24 -h retention freezing test but leaves post-shock freezing intact. The inability of intra-mPFC muscimol administration to impair contextual fear expression in post-shock freezing tests (Experiment 2b or 4) might suggest differential involvement of brain structures supporting contextual fear expression at different retention intervals (immediate post-shock versus long-term retention). Accordingly, freezing immediately after context-shock training may only require connectivity between the amygdala and periaqueductal gray (Herry and Johansen 2014). Our results could also be explained by the mPFC being required for the retrieval of a context-shock association. In adult mice, the mPFC, particularly the anterior cingulate (AC), has a role in the retrieval of remote but not recent contextual fear memories (Frankland et al. 2004; Frankland and Bontempi 2005). While we cannot rule out retrieval processes without additional studies, Experiment 3 suggests that inactivation of the mPFC on the testing day of the CPFE disrupts contextual fear expression (see Conclusion for additional proposed tests). Additionally, immediate expression of acquired fear may be supported by separate neural circuitry than fear expressed to a retrieved fear memory (Giustino and Maren 2015).

Several reports have suggested that pharmacological disruption of mPFC prior to foreground contextual fear conditioning (i.e., when the context is the sole predictor of the shock) has no effect on contextual fear acquisition (Holson and Walker 1986; Corcoran and Quirk 2007; Gilmartin et al. 2014; Giustino and Maren 2015). Accordingly, inactivation of the mPFC prior to foreground context-shock training in sCFC and the CPFE had no effect on acquisition of a context-shock association (Experiment $2 \mathrm{~b}$ and 4). One potential caveat is that, although animals receiving PBS or Muscimol acquired contextual fear at levels above nonassociative ISD control animals, levels of freezing were low during the retention test compared with the post-shock test. It is possible that the effect of intra-mPFC Muscimol administration on retention of contextual fear in sCFC is related to differences in freezing between these tests. This is unlikely as weaker forms of contextual fear conditioning (e.g., background context conditioning in auditory or trace fear conditioning) are susceptible to pharmacological disruption of the mPFC (Gilmartin et al. 2014; Giustino and Maren 2015). Importantly, Experiment 1 demonstrated that temporally separating learning about the context and context-shock association by $24 \mathrm{~h}$ necessitated the role of the mPFC during contextual learning in the CPFE unlike the concurrent context and contextshock learning that occurs in sCFC. One key difference between sCFC and the CPFE is that learning in sCFC can be supported by an individual feature-based neocortical system or by a hippocampal system in which the individual features of the context are bound into a conjunctive representation (Rudy and O'Reilly 2001; Wiltgen et al. 2006). Consequently, singularly disrupting the $\mathrm{mPFC}$ or dHPC does not eliminate conditioning in sCFC as compensatory hippocampal-amygdala or altered prefrontalamygdala systems may act to support learning, respectively (Wiltgen et al. 2006; Zelikowsky et al. 2012, 2013). In contrast to SCFC, learning in the CPFE can only be supported by a hippocampal-based conjunctive system (Rudy 2009; Schiffino et al. 2011; Jablonski et al. 2012) or perhaps a prefrontal-hippocampal interaction (Experiment 1). Future experiments manipulating features of the context to determine the type of context learning used in SCFC after regional inactivation of either DHPC or mPFC are needed to answer these questions. Nevertheless, our results demonstrate that temporally separating learning about the context and the context-shock association necessitates involvement of the medial prefrontal cortex during context learning in foreground contextual fear conditioning.

The current study examined the involvement of the mPFC across variants of contextual fear conditioning in adolescent rats (PD31). This period (adolescence) is marked by a relatively functionally mature $\mathrm{mPFC}$ in supporting fear behavior. The 
cytoarchitectonic and neurophysiological properties of the mPFC develop rapidly over the first 3 wk of life in the rat (PD1-PD21) (Van Eden and Uylings 1985; Zhang 2004). The transition from adolescence to adulthood is accompanied by an overall decrease in mPFC volume, reflective of extensive synaptic pruning coupled with small, targeted increases in dendritic complexity (Koss et al. 2014; Ferguson and Gao 2015; Drzewiecki et al. 2016). It is possible that the current results reflect a sensitive period in which the $\mathrm{mPFC}$, in tandem with the hippocampus, is recruited for context learning. Given that the hippocampus matures and begins supporting spatial learning and consolidation by the beginning of the fourth week of life in the rat (Rudy and Morledge 1994; Rudy 2009; Schiffino et al. 2011; Travaglia et al. 2016), it is unclear why the mPFC would be selectively recruited during this period but not later in development. We therefore predict that the same mPFC inactivation in adulthood would produce similar disruptions in context learning within the CPFE. Interestingly, the mPFC matures to support (and becomes necessary for) extinction learning and fear expression between PD17 and PD24 (Kim et al. 2009; Li et al. 2012). This corresponds to the same timeline in which the CPFE develops in the rat (PD17-PD24; Schiffino et al. 2011). Thus, in addition to hippocampal maturation, the development of the CPFE could reflect MPFC maturation and recruitment for conjunctive context learning. These are all fruitful avenues for future research.

The current set of experiments is the first demonstration that the mPFC is functionally required for initial context learning to acquire a context representation in the absence of aversive (emotional) stimuli. These findings are in contrast to the current view that the mPFC is not involved in initial fear or context learning, per se, but is mainly involved in regulation of fear expression and long-term systems consolidation of memory. Importantly, these findings allow us to draw conclusions about the changing role of the mPFC across phases of the CPFE. We propose that mPFC interacts with PHR and HPC to form a conjunctive context representation on the preexposure day. On the training day, this role of mPFC shifts to reconsolidation or updating of the conjunctive context representation to include shock as a feature, but not context retrieval or context-shock learning. On the test day, the mPFC modulates expression of contextual fear memory. Inclusion of a post-shock freezing measure following intracranial drug infusion on the training day of the CPFE allowed the current study to rule out acquisition processes in favor of processes related to memory consolidation. This novel working hypothesis concerning the changing role of mPFC across phases of the CPFE requires further investigation. Several predictions remain to be tested, including: (1) mPFC-HPC connectivity is critical for context learning during CPFE (mPFC-HPC "disconnection" performed on the preexposure day will disrupt the CPFE); (2) MPFC inactivation or protein synthesis inhibition immediately following immediateshock training should produce the same effects that occur with pretraining inactivation (Experiment 2); and (3) mPFC participates in expression but not retrieval on the testing day, that is, reminder treatments designed to facilitate retrieval would not reverse impaired freezing produced by $\mathrm{mPFC}$ inactivation (shown in Experiment 3). Future research with the CPFE paradigm promises to shed further light on the poorly understood role of mPFC in context and contextual fear learning and memory.

\section{Materials and Methods}

\section{Subjects}

Animal husbandry was as described in our previous reports (Heroux et al. 2016; Robinson-Drummer et al. 2016). There were a total of 223 adolescent Long-Evans rats (110 females and 113 males) split across four experiments, derived from 48 separate litters bred at the Office of Laboratory Animal Medicine at the University of Delaware. Time-mated females were housed with breeder males overnight and, if an ejaculatory plug was found the following morning that day was designated as gestational day (GD) 0 . Dams were housed in clear polypropylene cages measuring $45 \mathrm{~cm} \times 24 \mathrm{~cm} \times 21 \mathrm{~cm}$ with standard bedding and access to ad libitum water and rat chow. Animals were maintained on a 12:12 h light-dark cycle with lights on at 7:00 a.m. Date of birth was designated as postnatal day (PD) 0 . Litters were culled on PD3 to eight pups (usually four males and four females) and were paw-marked with subcutaneous injections of nontoxic black ink for later identification. Pups were weaned from their mother on PD21 and housed with same-sex litter mates in $45 \mathrm{~cm} \times$ $24 \mathrm{~cm} \times 17 \mathrm{~cm}$ cages. On PD29 animals were individually housed after stereotaxic surgery in small white polypropylene cages $(24 \mathrm{~cm} \times 18 \mathrm{~cm} \times 13 \mathrm{~cm})$ with ad libitum access to water and rat chow for the remainder of the experiment. All procedures were approved by the Institutional Animal Care and Use Committee at the University of Delaware following guidelines established by the National Institute of Health.

\section{Stereotaxic surgery}

Surgical implantation of intracranial injection cannulas in juvenile and adolescent rats has been previously described for intra-hippocampal and intra-mPFC cannula in our laboratory (Jablonski et al. 2010; Schiffino et al. 2011; Robinson-Drummer et al. 2016). Rats were obtained from post-weaning group housing on the morning of PD29 and anesthetized with a primary injection volume of $1 \mathrm{mg} / \mathrm{kg}$ of an 85:15 ketamine/xylazine drug mixture prior to surgery, with small supplemental doses given as needed. Guide cannulas (Plastics One) were bilaterally implanted to terminate in the mPFC using the following coordinates: anteroposterior (AP) $+9.0 \mathrm{~mm}$, mediolateral (ML), $\pm 0.6 \mathrm{~mm}$ relative to interaural midline, and dorsoventral (DV), $-2.3 \mathrm{~mm}$ relative to the top of the skull. Cannula were fixed in place on the skull using dental acrylic and curved "skull hooks" as previously reported (Schiffino et al. 2011; Robinson-Drummer et al. 2016). Following surgery, a dummy injector extending the same length as the drug injector tips (i.e., 1-mm extension) and dust caps were inserted in the guide cannula to reduce occlusion of the guide cannula. Rats were allowed to recover in individual white cages with electric heating pads placed under half of the cage floor. Twenty-four hours following surgery (PD30), animals were infused with $0.25 \mu \mathrm{L}$ of the vehicle phosphate buffered saline (PBS) in both hemispheres to reduce occlusion in the guide cannula and to acclimate the animals to being handled during infusions before the start of behavioral procedures the following day (PD31).

\section{Drug infusion}

Microinjections of the vehicle $\mathrm{PBS}$ or the $\mathrm{GABA}_{\mathrm{A}}$ receptor agonist muscimol (Sigma-Aldrich) were administered $\sim 15 \mathrm{~min}$ prior to behavioral procedures on either PD31 (Experiments 1 and 4), PD32 (Experiment 2), or PD33 (Experiment 3). Animals were briefly transported into a room directly adjacent to the colony room for drug infusion. Animals were gently held by hand while PBS or muscimol ( $2 \mu \mathrm{g} / \mu \mathrm{L}$ dissolved in PBS) was infused into both hemispheres at a rate of $0.25 \mu \mathrm{L} / \mathrm{min}$ for a single minute, resulting in a final infusion volume of $0.25 \mu \mathrm{L}$ and a final dose of $0.5 \mu \mathrm{g}$ per side for each animal. This dose was chosen in part because of its efficacy to disrupt hippocampal functioning given prior to any phase of the CPFE (Matus-Amat et al. 2004). This dose also inactivates the mPFC (Jo et al. 2007; Maeng et al. 2010). Injector tips were left in the guide cannula for $1 \mathrm{~min}$ following infusion to allow sufficient diffusion of the drug. Animals were returned to their home-cage for $\sim 15$ min until the start of behavioral testing.

\section{Apparatus and stimuli}

The apparatus and stimuli used have been previously described (Heroux et al. 2016; Murawski and Stanton 2010; Murawski 
et al., 2012; Robinson-Drummer et al. 2016). Fear conditioning occurred in four Plexiglas chambers measuring $16.5 \mathrm{~cm} \times 12.1$ $\mathrm{cm} \times 21.6 \mathrm{~cm}$ which were arranged in a $2 \times 2$ formation on a Plexiglas stand within a fume hood to provide ambient light and background noise (Context A). Each chamber had a grid floor made of nine stainless steel bars $(11.5 \mathrm{~cm}$ from the top of the chamber), $0.5 \mathrm{~cm}$ in diameter and spaced $1.25 \mathrm{~cm}$ apart. The alternate context (Context B) consisted of the same Plexiglas chambers with a convex wire mesh insert that covered the back wall and floor of the chamber and a white paper sleeve that covered the outside walls of the chamber. The 2-sec, 1.5-mA footshock unconditioned stimulus (US) was delivered using a shock scrambler (VT ENV-414S, Med Associates) connected to the grid floor of the chamber. The fear chambers were cleaned with 5\% ammonium hydroxide solution prior to each load of experimental animals. Videos of each session (preexposure, training, testing) were recorded using Freeze Frame 3.0 software (Actimetrics) with freezing defined as a bout of $0.75 \mathrm{sec}$ or longer without a change in video pixilation.

\section{Context preexposure facilitation effect (Experiments 1-3)}

The multiple preexposure CPFE behavioral procedure has been described previously (Dokovna et al. 2013; Heroux et al. 2016; Robinson-Drummer et al. 2016). The CPFE procedure took place over the course of $3 \mathrm{~d}$ from PD31 to PD33 ( $\pm 1 \mathrm{~d})$. Animals were assigned to either preexposure (Precondition) or alternate preexposure (Alt Pre condition). Animals in the preexposure group were preexposed to the training context (Context A), and animals in the Alt Pre group were preexposed to the alternate context (Context B, as described by Murawski and Stanton 2010). Animals preexposed to an alternate context (Context B) on the first day of the CPFE serve as nonassociative behavioral controls as they fail to acquire a context representation needed to be retrieved and associated with shock on the training day (Rudy 2009). Multiple preexposure consisted of one initial 5-min exposure to the chamber, followed by five 1-min exposures, with a 1-min interval between exposures. Animals were placed in transport boxes on a cart inside the training room during the 1-min inter-trial interval.

On PD31, animals were weighed and carted to the behavioral testing room in transport cages of clear Lexan $(11 \mathrm{~cm} \times 11 \mathrm{~cm} \times 18$ $\mathrm{cm})$ covered on all sides with orange construction paper to obscure visual cues during transport. Pre animals were placed in Context A for the multiple preexposure, whereas animals in the Alt Pre group underwent multiple preexposure in the alternate context (Context B). On PD32, single rats were carried into the testing room, placed in their respective training chamber, and given two immediate (i.e., occurring $<5 \mathrm{sec}$ upon placement) $1.5-\mathrm{mA}, 2$-sec footshocks separated by $1 \mathrm{sec}$ in Context A. Animals were immediately removed from the chambers following the footshocks, returned to their transport cages, and then taken back to their home cages. In Experiment $2 \mathrm{~b}$, the rats were left in the training chambers following the two immediate shocks for a 3-min postshock freezing test consisting of no additional shock presentations. On PD33, animals were tested in Context A for $5 \mathrm{~min}$ in the same chamber in which they were trained. Testing consisted of a 5-min exposure to the chamber with no additional exposure to the unconditioned stimulus. Drug infusions of PBS or muscimol took place $15 \mathrm{~min}$ before multiple preexposure on PD31 (Experiment 1 ), before immediate shock training on PD32 (Experiments 2a and $2 \mathrm{~b}$ ), or before retention testing on PD33 (Experiment 3).

\section{Standard contextual fear conditioning (Experiment 4)}

The sCFC procedure has been described previously (Malkani and Rosen 2001; Schreiber et al. 2014). The sCFC procedure took place over the course of $2 \mathrm{~d}$ from PD31 to PD32 ( $\pm 1 \mathrm{~d})$. All chambers, stimuli, and drug infusion protocols used were identical to the ones used in Context A for the CPFE experiments (see Apparatus and stimuli and drug infusion). On PD31, animals were assigned to one of four behavior conditions: Post-shock, Retention, Immediate-shock-post-shock (Imm-Shock Post-shock), and Immediate-shock-retention (Imm-Shock Retention). Animals in the Post-shock and Retention conditions both received $3 \mathrm{~min}$ of context exposure in Context A, followed by two 1.5-mA, 2-sec footshocks separated by $1 \mathrm{sec}$. Subsequently, animals in the Post-shock condition received three additional minutes of exposure to the chamber with no additional shock presentations whereas animals in the Retention condition were returned to their home cages immediately after the shock training. Animals in both of the Imm-Shock conditions were given two footshocks without any context exposure. These groups served as behavioral controls for their respective delayed-shock conditions as the placementto-shock interval was under $5 \mathrm{sec}$ resulting in the immediate shock deficit (i.e., an inability to form a context-shock association due to no prior context exposure) (Fanselow 1990). On PD32, rats in both retention conditions were tested in Context A for $5 \mathrm{~min}$ in the same chamber they had been trained in with no additional presentations of the unconditioned stimulus.

\section{Histology}

Within $24-48 \mathrm{~h}$ of behavioral testing, rats were sacrificed by rapid decapitation. Brains were removed and frozen in $-45^{\circ} \mathrm{C}$ isopentane and then stored at $-80^{\circ} \mathrm{C}$ until being sectioned on a microtome. Coronal sections of $\sim 40 \mu \mathrm{m}$ were taken throughout the entirety of the cannula tracts visible in the brain tissue. The $40 \mu \mathrm{m}$ coronal slices were mounted on charged microscope slides and stained with Neutral Red (1\%). Slides were photocaptured and analyzed to confirm the placement of the cannula injector tip in the mPFC. Out of 223 surgeries (see Fig. 1A for placements), there were a total of 10 misplaced cannula with the distribution as follows: Experiment $1(n=4)$, Experiment $2(n=2)$, Experiment 3 $(n=1)$, and Experiment $4(n=3)$.

A subset of animals underwent the same surgical and cannula clearing procedures mentioned previously (see Stereotaxic surgery) and were used to visualize the spread of muscimol in the mPFC. Rats were infused with fluorophore-conjugated muscimol (Muscimol BODIPY-TMR-X, Fisher Scientific) at the same volume, dose, and infusion rate mentioned previously (see Drug Infusion) (Allen et al. 2008). The fluorescent muscimol was diluted to a concentration of $2 \mu \mathrm{g} / \mu \mathrm{L}$ into a solution made of half PBS and half DMSO to ensure dissolution (Hallock et al. 2013). The animals were sacrificed by rapid decapitation 15 min after infusion of the fluorescent muscimol in order to match the spread to what the experimental animals received immediately prior to behavioral testing. The brains were removed and flash frozen in $-45^{\circ} \mathrm{C}$ isopentane, stored at $-80^{\circ} \mathrm{C}$, and then coronal slices were sectioned at $60 \mu \mathrm{m}$. The slices were mounted on charged microscope slides and counterstained with ProLong Diamond with DAPI (Life Technologies). The stained slices incubated in a cool, dark room at room temperature for $3 \mathrm{~d}$ before being visualized with a confocal microscope (ZEISS LSM 880) in the Bioimaging Center at the Delaware Biotechnology Institute. A digital plate from the Paxinos and Watson (2007) rat brain atlas was overlaid on the image to visualize the spread (see Fig. 1B).

\section{Data analysis}

Data processing procedures have been described previously (Heroux et al. 2016; Robinson-Drummer et al. 2016). A human observer blind to the experimental groups verified the freezing threshold setting with Freeze View 3.0 (Actimetrics). The software program computes a "motion index" that was adjusted to set a freezing threshold separately for each animal (per software instructions) by a blind observer who verified from the video record whether or not small movements were scored as freezing. Once set, the threshold did not change during a session. We have validated this procedure against other scoring methods (e.g., hand scoring of video records by two blind observers). Freezing behavior was scored as the total percent time spent freezing (defined as the cessation of all movement except breathing) in each respective session bin (context exposure, preshock freezing, post-shock freezing, and a 24 -h retention test).

Once percent freezing was reliably determined, the data were imported into STATISTICA 64 data analysis software and freezing behavior was analyzed with a series of ANOVAs. Statistical 
significance was set to $P<0.05$. For Experiments $1-3$, a "Pooled Alt-Pre" condition was used as reported previously (Heroux et al. 2016; Robinson-Drummer et al. 2016). Data from animals in the nonassociative alternate preexposure group were collapsed across drug condition as freezing was uniformly low and there were no significant differences between control animals given either drug $(P s>0.10)$. This reduces animal use and simplifies the experimental design. The unpooled Alt-Pre freezing data for each experiment is as follows (AVG \pm SEM): Expt.1 (Alt-Pre-Saline: $6.57 \pm 3.21$; Alt-Pre-Muscimol: $8.70 \pm 4.85$ ), Expt.2a (Alt-PreSaline: $8.55 \pm 3.41$; Alt-Pre-Muscimol: $11.01 \pm 4.00)$; Expt.2b PostShock (Alt-Pre-Saline: $15.20 \pm 2.65$; Alt-Pre-Muscimol: 16.10 \pm 4.26 ); Expt.2b Retention (Alt-Pre-Saline: $9.06 \pm 1.51$; Alt-Pre-Muscimol: $5.63 \pm 2.03$ ); Expt.3 (Alt-Pre-Saline: $16.26 \pm 2.45$; Alt-Pre-Muscimol: $19.25 \pm 5.58)$. There were also no main effects or interactions involving sex across any of the experiments $(P \mathrm{~s}>0.05)$, so the data were collapsed across this variable.

Freezing behavior for Experiments 1, 2a, and 3 was analyzed with a one-way ANOVA (Drug; PBS, Muscimol, Pooled-Alt-Pre). Freezing behavior for Experiment $2 \mathrm{~b}$ was analyzed with a Drug (PBS, Muscimol, Pooled-Alt-Pre) $\times$ Phase (Post-shock, Retention) repeated-measures ANOVA with Phase being the repeated measure. Freezing behavior for Experiment 4 was analyzed with a Drug $(\mathrm{PBS}$, Muscimol) $\times$ Phase (Post-shock freezing, Retention freezing) $\times$ Training (Imm-Shock, Delayed-Shock) three-way factorial ANOVA with only between-groups factors. Post hoc contrasts were performed with Newman-Keuls tests. Consistent with our previous reports (Schiffino et al. 2011; Heroux et al. 2016; Robinson-Drummer et al. 2016), a rat was excluded from analysis as an outlier if it had a score of \pm 1.96 standard deviations from its group mean, however, the average $Z$-score of removed outliers averaged across all experiments (1-4) was \pm 3.01 ( \pm 0.32 SEM). The outliers were distributed as follows: three rats were excluded from analysis as outliers in Experiment 1 (Pre-PBS $=2$, Pooled-AltPre $=1) ; 5$ rats in Experiment 2A (Pre-PBS $=2$, Pre-Muscimol =2, Pooled-Alt-Pre $=1$ ); 3 rats in Experiment 2B (Pre-Muscimol $=2$; Pooled-Alt-Pre $=1$ ); 3 rats in Experiment 3 (Pre-PBS $=1$, PooledAlt-Pre $=2$ ); and, 2 rats in Experiment 4 (Retention-PBS=1, Retention-Muscimol =1).

\section{Acknowledgments}

These experiments were supported by the University of Delaware and NIH grant 1-R01-HD075066-01A1 to M.E.S. and J.B.R.; and R01-MH106553-01A1 to Jaclyn Schwarz. Imaging was completed using the ZEISS LSM 880 confocal microscope in the Bioimaging Center at the Delaware Biotechnology Institute (Newark, Delaware), supported by NIH grant 1-S10-OD016361 and INBRE grant P20GM103446. We appreciate the technical contribution of Katelyn $\mathrm{N}$. Buban for assistance with histology and surgery.

\section{References}

Allen TA, Narayanan NS, Kholodar-Smith DB, Zhao Y, Laubach M, Brown TH. 2008. Imaging the spread of reversible brain inactivations using fluorescent muscimol. J Neurosci Methods 171: 30-38.

Asok A, Schreiber WB, Jablonski SA, Rosen JB, Stanton ME. 2013. Egr-1 increases in the prefrontal cortex following training in the context preexposure facilitation effect (CPFE) paradigm. Neurobiol Learn Mem 106: $145-153$.

Bero AW, Meng J, Cho S, Shen AH, Canter RG, Ericsson M, Tsai L-H. 2014 Early remodeling of the neocortex upon episodic memory encoding. Proc Natl Acad Sci 111: 11852-11857.

Burman MA, Murawski NJ, Schiffino FL, Rosen JB, Stanton ME. 2009. Factors governing single-trial contextual fear conditioning in the weanling rat. Behav Neurosci 123: 1148-1152.

Burton BG, Hok V, Save E, Poucet B. 2009. Lesion of the ventral and intermediate hippocampus abolishes anticipatory activity in the medial prefrontal cortex of the rat. Behav Brain Res 199: 222-234.

Chakraborty T, Asok A, Stanton ME, Rosen JB. 2016. Variants of contextual fear conditioning induce differential patterns of Egr-1 activity within the young adult prefrontal cortex. Behav Brain Res 302: 122-130.

Corcoran KA, Quirk GJ. 2007. Activity in prelimbic cortex is necessary for the expression of learned, but not innate, fears. J Neurosci 27: 840-844.
Dokovna LB, Jablonski SA, Stanton ME. 2013. Neonatal alcohol exposure impairs contextual fear conditioning in juvenile rats by disrupting cholinergic function. Behav Brain Res 248: 114-120.

Drzewiecki CM, Willing J, Juraska JM. 2016. Synaptic number changes in the medial prefrontal cortex across adolescence in male and female rats: a role for pubertal onset. Synapse 70: $361-368$.

Einarsson EÖ, Nader K. 2012. Involvement of the anterior cingulate cortex in formation, consolidation, and reconsolidation of recent and remote contextual fear memory. Learn Mem 19: 449-452.

Fanselow MS. 1980. Conditional and unconditional components of post shock freezing. Pavlov J Biol Sci 15: 177-182.

Fanselow MS. 1990. Factors governing one-trial contextual conditioning. Anim Learn Behav 18: 264-270.

Fanselow MS, Poulos AM. 2005. The neuroscience of mammalian associative learning. Annu Rev Psychol 56: 207-234.

Ferguson BR, Gao WJ. 2015. Development of thalamocortical connections between the mediodorsal thalamus and the prefrontal cortex and its implication in cognition. Front Hum Neurosci 8: 1027.

Frankland PW, Bontempi B. 2005. The organization of recent and remote memories. Nat Rev Neurosci 6: 119-130.

Frankland PW, Bontempi B, Talkton LE, Kaczmarek L, Silva AJ. 2004. The involvement of the anterior cingulate cortex in remote contextual fear memory. Science 304: 881-883.

Frankland PW, Ding H, Takahashi E, Suzuki A, Kida S, Silva AJ. 2006. Stability of recent and remote contextual fear memory. Learn Mem 13: 451-457.

Gilmartin MR, Kwapis JL, Helmstetter FJ. 2012. Trace and contextual fear conditioning are impaired following unilateral microinjection of muscimol in the ventral hippocampus or amygdala, but not the medial prefrontal cortex. Neurobiol Learn Mem 97: 452-464.

Gilmartin MR, Kwapis JL, Helmstetter FJ. 2013. NR2A- and NR2B-containing NMDA receptors in the prelimbic medial prefrontal cortex differentially mediate trace, delay, and contextual fear conditioning. Learn Mem 20: 290-294.

Gilmartin MR, Balderston NL, Helmstetter FJ. 2014. Prefrontal cortical regulation of fear learning. Trends Neurosci 37: 445-464.

Giustino TF, Maren S. 2015. The role of the medial prefrontal cortex in the conditioning and extinction of fear. Front Behav Neurosci 9: 298.

Giustino TF, Fitzgerald PJ, Maren S. 2016. Fear expression suppresses medial prefrontal cortical firing in rats. PLoS One 11: e0165256.

Hallock HL, Wang A, Shaw CL, Griffin AL. 2013. Transient inactivation of the thalamic nucleus reuniens and rhomboid nucleus produces deficits of a working-memory dependent tactile-visual conditional discrimination task. Behav Neurosci 127: 860-866.

Heroux NA, Robinson-Drummer PA, Rosen JB, Stanton ME. 2016. NMDA receptor antagonism disrupts acquisition and retention of the context preexposure facilitation effect in adolescent rats. Behav Brain Res 301: 168-177.

Herry C, Johansen J. 2014. Encoding of fear learning and memory in distributed neuronal circuits. Nat Neurosci 17: 1644-1654.

Hok V, Save E, Lenck-Santini PP, Poucet B. 2005. Coding for spatial goals in the prelimbic/infralimbic area of the rat frontal cortex. Proc Natl Acad Sci 102: 4602-4607.

Hok V, Chah E, Save E, Poucet B. 2013. Prefrontal cortex focally modulates hippocampal place cell firing patterns. J Neurosci 33: 3443-3451.

Holson RR, Walker C. 1986. Mesial prefrontal cortical lesions and timidity in rats. II. Reactivity to novel stimuli. Physiol Behav 37: 231-238.

Jablonski SA, Watson DJ, Stanton ME. 2010. Role of medial prefrontal NMDA receptors in spatial delayed alternation in 19-, 26-, and 33-day-old rats. Dev Psychobiol 52: 583-591.

Jablonski SA, Schiffino FL, Stanton ME. 2012. Role of age, post-training consolidation, and conjunctive associations in the ontogeny of the context preexposure facilitation effect. Dev Psychobiol 54: 714-722.

Ji G, Neugebauer V. 2012. Modulation of medial prefrontal cortical activity using in vivo recordings and optogenetics. Mol Brain 5: 36.

Jin J, Maren S. 2015. Prefrontal-hippocampal interactions in memory and emotion. Front Syst Neurosci 9: 170.

Jo YS, Park EH, Kim IH, Park SK, Kim H, Kim HT, Choi JS. 2007. The medial prefrontal cortex is involved in spatial memory retrieval under partial-cue conditions. J Neurosci 27: 13567-13578.

Kim JJ, Rison RA, Fanselow MS. 1993. Effects of amygdala, hippocampus, and periaqueductal gray lesions on short- and long-term contextual fear. Behav Neurosci 107: 1093-1098.

Kim JH, Hamlin AS, Richardson R. 2009. Fear extinction across development: the involvement of the medial prefrontal cortex as assessed by temporary inactivation and immunohistochemistry. I Neurosci 29: 10802-10808.

Kitamura T, Inokuchi K. 2014. Role of adult neurogenesis in hippocampal-cortical memory consolidation. Mol Brain 7: 13.

Koss WA, Belden CE, Hristov AD, Juraska JM. 2014. Dendritic remodeling in the adolescent medial prefrontal cortex and the basolateral amygdala of male and female rats. Synapse 68: 61-72. 
Laurent V, Westbrook RF. 2009. Inactivation of the infralimbic but not the prelimbic cortex impairs consolidation and retrieval of fear extinction. Learn Mem 16: 520-529.

Lee JL. 2010. Memory reconsolidation mediates the updating of hippocampal memory content. Front Behav Neurosci 4: 168.

Li S, Kim JH, Richardson R. 2012. Differential involvement of the medial prefrontal cortex in the expression of learned fear across development. Behav Neurosci 126: 217-225.

Maddox SA, Monsey MS, Schafe GE. 2011. Early growth response gene 1 (Egr-1) is required for new and reactivated fear memories in the lateral amygdala. Learn Mem 18: 24-38.

Maeng LY, Waddell J, Shors TJ. 2010. The prefrontal cortex communicates with the amygdala to impair learning after acute stress in females but not in males. J Neurosci 30: 16188-16196.

Malkani S, Rosen JB. 2001. N-Methyl-D-aspartate receptor antagonism blocks contextual fear conditioning and differentially regulates early growth response-1 messenger RNA expression in the amygdala: implications for a functional amygdaloid circuit of fear. Neuroscience 102: $853-861$.

Malkani S, Wallace KJ, Donley MP, Rosen JB. 2004. An egr-1 (zif268) antisense oligodeoxynucleotide infused into the amygdala disrupts fear conditioning. Learn Mem 11: 617-624.

Maren S, Aharonov G, Stote DL, Fanselow MS. 1996. N-methyl-D-aspartate receptors in the basolateral amygdala are required for both acquisition and expression of conditional fear in rats. Behav Neurosci 110: 1365-1374.

Matus-Amat P, Higgins EA, Barrientos RM, Rudy JW. 2004. The role of the dorsal hippocampus in the acquisition and retrieval of context memory representations. J Neurosci 24: 2431-2439.

Matus-Amat P, Higgins EA, Sprunger D, Wright-Hardesty K, Rudy JW. 2007. The role of dorsal hippocampus and basolateral amygdala NMDA receptors in the acquisition and retrieval of context and contextual fear memories. Behav Neurosci 121: 721-731.

Murawski NJ, Stanton ME. 2010. Variants of contextual fear conditioning are differentially impaired in the juvenile rat by binge ethanol exposure on postnatal days 4-9. Behav Brain Res 212: 133-142.

Murawski NJ, Klintsova AY, Stanton ME. 2012. Neonatal alcohol exposure and the hippocampus in developing male rats: effects on behaviorally induced CA1 c-Fos expression, CA1 pyramidal cell number, and contextual fear conditioning. Neuroscience 206: 89-99.

Paxinos G, Watson C. 2007. The rat brain in stereotaxic coordinates, 6 th ed. Academic Press, New York, NY, pp. 50, 52, 54, and 56.

Quinn JJ, Ma QD, Tinsley MR, Koch C, Fanselow MS. 2008. Inverse temporal contributions of the dorsal hippocampus and medial prefrontal cortex to the expression of long-term fear memories. Learn Mem 15: 368-372.

Robinson-Drummer PA, Dokovna LB, Heroux NA, Stanton ME. 2016. Cholinergic mechanisms of the context preexposure facilitation effect in adolescent rats. Behav Neurosci 130: 196-205.

Rozeske RR, Valerio S, Chaudun F, Herry C. 2015. Prefrontal neuronal circuits of contextual fear conditioning. Genes Brain Behav 14: 22-36.

Rudy JW. 2009. Context representations, context functions, and the parahippocampal-hippocampal system. Learn Mem 16: 573-585.

Rudy JW, Morledge P. 1994. Ontogeny of contextual fear conditioning in rats: implications for consolidation, infantile amnesia, and hippocampal system function. Behav Neurosci 108: 227-234.
Rudy JW, O'Reilly RC. 2001. Conjunctive representations, the hippocampus, and contextual fear conditioning. Cogn Affect Behav Neurosci 1: 66-82.

Rudy JW, Wright-Hardesty K. 2005. The temporal dynamics of retention of a context memory: Something is missing. Learn Mem 12: 172-177.

Rudy JW, Huff NC, Matus-Amat P. 2004. Understanding contextual fear conditioning: Insights from a two-process model. Neurosci Biobehav Rev 28: $675-685$.

Schiffino FL, Murawski NJ, Rosen JB, Stanton ME. 2011. Ontogeny and neural substrates of the context preexposure facilitation effect. Neurobiol Learn Mem 95: 190-198.

Schreiber WB, Asok A, Jablonski SA, Rosen JB, Stanton ME. 2014. Egr-1 mRNA expression patterns in the prefrontal cortex, hippocampus, and amygdala during variants of contextual fear conditioning in adolescent rats. Brain Res 1576: 63-72.

Sierra-Mercado D, Padilla-Coreano N, Quirk GJ. 2011. Dissociable roles of prelimbic and infralimbic cortices, ventral hippocampus, and basolateral amygdala in the expression and extinction of conditioned fear. Neuropsychopharmacology 36: 529-538.

Squire LR, Alvarez P. 1995. Reterograde amnesia and memory consolidation: a neurobiological perspective. Curr Opin Neurobiol 5: 169-177.

Stern CA, Gazarini L, Vanvossen AC, Hames MS, Bertoglio LJ. 2014. Activity in prelimbic cortex subserves fear memory reconsolidation over time. Learn Mem 21: 14-20.

Tovote P, Fadok JP, Lüthi A. 2015. Neuronal circuits for fear and anxiety. Nat Rev Neurosci 16: 317-331.

Travaglia A, Bisaz R, Sweet ES, Blitzer R, Alberini CM. 2016. Infantile amnesia reflects a developmental critical period for hippocampal learning. Nat Neurosci 19: 1225-1233.

Van Eden CG, Uylings HB. 1985. Cytoarchitectonic development of the prefrontal cortex in the rat. J Comp Neurol 241: 253-267.

Wang SH, Morris RG. 2010. Hippocampal-neocortical interactions in memory formation, consolidation, and reconsolidation. Annu Rev Psychol 61: 49-79, C1-4.

Wiltgen BJ, Sanders MJ, Anagnostaras SG, Sage JR, Fanselow MS. 2006. Context fear learning in the absence of the hippocampus. J Neurosci 26: 5484-5491.

Zelikowsky M, Bissiere S, Fanselow MS. 2012. Contextual fear memories formed in the absence of the dorsal hippocampus decay across time. I Neurosci 32: 3393-3397.

Zelikowsky M, Bissiere S, Hast TA, Bennett RZ, Abdipranoto A, Vissel B, Fanselow MS. 2013. Prefrontal microcircuit underlies contextual learning after hippocampal loss. Proc Natl Acad Sci 110: 9938-9943.

Zelikowsky M, Hersman S, Chawla MK, Barnes CA, Fanselow MS. 2014. Neuronal ensembles in amygdala, hippocampus, and prefrontal cortex track differential components of contextual fear. J Neurosci 34: 8462-8466.

Zhang ZW. 2004. Maturation of layer V pyramidal neurons in the rat prefrontal cortex: intrinsic properties and synaptic function. J Neurophysiol 91: 1171-1182.

Received February 10, 2017; accepted in revised form May 12, 2017. 


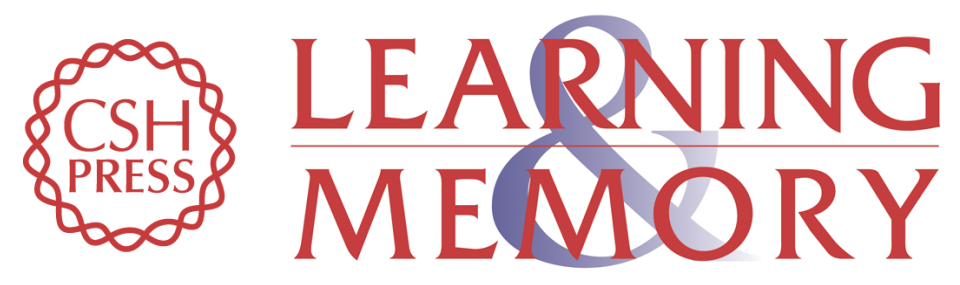

\section{Differential involvement of the medial prefrontal cortex across variants of contextual fear conditioning}

Nicholas A. Heroux, Patrese A. Robinson-Drummer, Hollie R. Sanders, et al.

Learn. Mem. 2017, 24:

Access the most recent version at doi:10.1101/Im.045286.117

References This article cites 71 articles, 22 of which can be accessed free at: http://learnmem.cshlp.org/content/24/8/322.full.html\#ref-list-1

Creative This article is distributed exclusively by Cold Spring Harbor Laboratory Press for the Commons License first 12 months after the full-issue publication date (see

http://learnmem.cshlp.org/site/misc/terms.xhtml). After 12 months, it is available under a Creative Commons License (Attribution-NonCommercial 4.0 International), as described at http://creativecommons.org/licenses/by-nc/4.0/.

Email Alerting Receive free email alerts when new articles cite this article - sign up in the box at the Service top right corner of the article or click here. 